\title{
McGill
}

\section{An MRI based average macaque monkey stereotaxic atlas and space (MNI monkey space)}

\author{
Stephen Frey, Deepak N. Pandya, M. Mallar Chakravarty, Lara \\ Bailey, Michael Petrides, D. Louis Collins \\ Neurolmage, Volume 55, Issue 4, 15 April 2011, Pages 1435-1442 \\ doi: 10.1016/j.neuroimage.2011.01.040 \\ http://www.sciencedirect.com/science/article/pii/S1053811911000759 \\ Copyright 2011 Elsevier Inc. This postprint provided under the terms of a \\ Creative Commons Attribution-NonCommercial-NoDerivatives license.
}




\title{
An MRI based average macaque monkey stereotaxic atlas and space (MNI monkey space)
}

\author{
Stephen Frey ${ }^{\mathrm{a}}$, Deepak N. Pandya ${ }^{\mathrm{b}}$, M. Mallar Chakravarty ${ }^{\mathrm{c}, \mathrm{d}}$, Lara Bailey ${ }^{\mathrm{a}}$, Michael \\ Petrides $^{\mathrm{a}}$, D. Louis Collins ${ }^{\mathrm{a}}$ \\ ${ }^{a}$ Montreal Neurological Institute, McGill University, Montreal, Quebec, Canada \\ ${ }^{\mathrm{b}}$ Department of Anatomy and Neurobiology, Boston University, Boston, Massachusetts, \\ USA \\ ${ }^{\mathrm{c}}$ Rotman Research Institute, Baycrest Hospital, Toronto, Ontario, Canada \\ ${ }^{\mathrm{d}}$ Mouse Imaging Center, Hospital for Sick Children, Toronto, Ontario, Canada
}

Total number of pages: 19

Total number of figures: 7

Total number of tables: 3

Abstract: 207

Total number of words: 4957

Key words: stereotactic, brain templates, unbiased brain atlas, three-dimensional, localization of function, brain anatomy, linear and nonlinear averages

Correspondence:

Stephen Frey Ph.D.

Cognitive Neuroscience Unit

Montreal Neurological Institute

3801 University Street

Montreal, Quebec

H4A2W5

Tel: 514-398-2579

Fax: 514-398-1338

email: stephen@bic.mni.mcgill.ca 


\begin{abstract}
In studies of the human brain, a standard stereotaxic space such as the Montreal Neurological Institute (MNI space) is widely used to provide a common reference for the three-dimensional localization of functional activation foci and anatomical structures, enabling the comparison of results obtained across different studies. Here we present a standard macaque monkey brain MRI template that offers a common stereotaxic reference frame to localize anatomical and functional information in an organized and reliable way for comparison across individual monkeys and studies. We have used MRI volumes from a group of 25 normal adult macaque monkeys (18 cynomolgus and 7 rhesus) to create a common standard macaque monkey brain as well as atlases for each of these species separately. In addition, the digital macaque monkey volume was subjected to $3 \mathrm{D}$ volumetric analysis and comparison of brain structures between the individual brains and the average atlas. Furthermore, we provide a means of transforming any macaque MRI volume into MNI monkey space coordinates in 3D using simple web based tools. Coordinates in MNI monkey space can also be transformed into the coordinate system of a detailed neuroanatomical paper atlas (Paxinos et al., 2008), enabling researchers to identify and delineate cortical and subcortical structures in their individual macaque monkey brains.
\end{abstract}




\section{Introduction}

A standard average template or brain atlas is commonly used in the human neuroimaging field to localize brain function and to provide a common method for reporting findings from different experiments. The original human standard proportional stereotaxic space, known as Talairach space (Talairach and Tournoux, 1988), was generated from a single cadaver brain. Image volumes can be transformed into this space through estimation of a piece-wise linear transformation procedure. The Montreal Neurological Institute (MNI) space (Evans et al., 1994a) redefined the standard proportional stereotaxic space used in the imaging community. This space was defined using the average of 305 normal anatomical MRI scans (MNI 305 template) that were scaled linearly (Collins et al., 1994) to landmarks within the Talairach atlas and is more representative of the human population. Since then, a further 152 normal MRI data sets have been transformed to the MNI 305 template (Evans et al., 1994a,b; Mazziotta et al., 2001; see Lancaster et al., 2007 for review) to create one of the most used human stereotaxic reference systems in the neurosciences at present (ICBM 152 template; http://www.bic.mni.mcgill.ca/ServicesAtlases/HomePage.

In order to elucidate human brain function, researchers have relied on comparative anatomical and functional studies of the nonhuman primate to answer neurological, functional, and anatomical questions. The localization and identification of structures in the nonhuman primate brain using a standardized or common coordinate system is greatly needed due to the increased importance of comparing the results from different studies and different populations. 
Two-dimensional macaque monkey atlases (Martin and Bowden, 1996; Paxinos et al., 2000, 2008; Saleem and Logothetis, 2006; Szabo and Cowan, 1984; Winters et al., 1969), with their own coordinate system and anatomical labels, are often used to corroborate findings from imaging studies. Stereotaxic paper atlases or stereotaxic coordinates from the interaural plane have also been used to guide placement of electrodes, cannulae or other probes into the brain for use in electrophysiological, or anatomical tracer injection studies, as well as making lesion in particular parts of the brain (Hampson et al., 2004; Lévesque and Parent, 2005; Saunders et al., 1990; Malkova et al., 1997; Murray and Mishkin, 1998). However, the extrapolation of 2D information from a particular atlas to a given animal's brain without neuronavigation techniques or registration procedures (Frey et al., 2004) is often difficult to accomplish. Furthermore, there is considerable variability across individual monkey brains and it is essential to have a method for locating anatomical differences amongst animals (functional or structural) while simultaneously preserving the established relationship between brain structures. To address these needs, digital macaque probabilistic atlases were created and are available on the internet (Black et al., 2001; Frey et al., 2006; 2009; Vincent et al., 2007; McLaren et al., 2009; see Van Essen and Dierker, 2007 for review). Only recently have there been means of transforming monkey MRI coordinates to macaque monkey paper atlases (Chakravarty et al., 2008; McLaren et al., 2009), although a more userfriendly method of transforming these stereotaxic data sets is needed. Our goal was to create a nonlinear image template to which new image volumes could be easily matched using fully automated, unbiased, nonlinear registration methods. It is for this reason that we present an unbiased monkey atlas that does not rely on the anatomy of a single 
subject, but instead depends on nonlinear normalization (Collins et al., 1995; Collins and Evans, 1997) of numerous macaque monkey brains mapped to an average template image that is faithful to the location of anatomical structures. This 3D atlas is of particular interest to researchers who need to quantify, locate or screen for anatomical and structural differences in a single animal's brain in a systematic and efficient manner.

Also presented here are classification techniques that have been applied to the average macaque atlas in order to identify and quantify tissue types such as gray and white matter as well as cerebral spinal fluid (CSF) of all the MRI volumes and the average macaque atlas. Manual anatomical delineation of certain brain structures (i.e. the medial temporal lobe structures) on the average macaque template have been included to help visualize annotated 3D identification of certain regions and their spatial relationship with each other. Furthermore, deformation maps have been included to demonstrate the patterns of geometrical variability inherent in all the monkey brains when registered with the average brain through nonlinear transformation. Finally, in addition to the average macaque atlas, we present $3 \mathrm{D}$ templates of both the rhesus macaque and the cynomolgus macaque monkey in order to look at the similarities and differences of the two species.

\section{Materials and methods}

\section{Subjects}

The subjects were 25 normal adult macaque monkeys (18 cynomolgus; Macaca fascicularis [3 females], and 7 rhesus; Macaca mulatta [2 female]). The weights of the animals were approximately 3.3 to $13.1 \mathrm{~kg}($ mean $=6.5, \mathrm{sd}=2.1)$ at the time of MRI. The protocols used were approved by the Montreal Neurological Institute Animal Ethics 
Committee and conformed to the Canadian Council of Animal Care guidelines for the humane care of laboratory animals.

\section{MRI scanning sessions}

MRI data was acquired on a Siemens Sonata 1.5T magnet in the context of stereotaxic surgery planning with a 3D transverse T1w acquisition. Each monkey was initially tranquilized with Ketamine $\left(10 \mathrm{mg} / \mathrm{kg}\right.$, Ketaset $\left.{ }^{\circledR}\right)$ and acepromazine $(0.4 \mathrm{mg} / \mathrm{kg}$, im, Atravet $\left.{ }^{\circledR}\right)$, and deep anesthesia was maintained with isoflurane (3-4\% at induction and $0.8-2 \%$, Isoflo®) throughout the procedure. The monkey was then placed in the magnet in the standard supine position. Throughout the scan, the monkey's oxygen saturation levels $\left(\mathrm{SPO}_{2}\right)$ and heart rate were monitored using pulse oximetry with an infrared sensor that was clipped to the animal's hallux through a fiber optic cable that was attached to a monitor. Data were acquired with different voxels sizes as the $\mathrm{T} 1$ sequence was optimized over time, finally settling on a $320 \times 320$ matrix with $0.60 \times 0.60 \times 0.60 \mathrm{~mm}$ voxels $(104$ slices, $\mathrm{TR}=22 \mathrm{~ms}, \mathrm{TE}=9.5 \mathrm{~ms}, \mathrm{FOV}=192)$. Please refer to Table 1 in the supplementary information for the different imaging parameters. In two cynomolgus monkey scans several slices from the temporal lobes were excluded due to a misaligned field of view and therefore these scans two were not included in the volumetric analyses.

\section{MRI image registration}

Image pre-processing included non-uniform intensity correction (Sled et. al., 1998) and intensity normalization to a range of $0-100$. All data was stored with floating-point voxels to minimize loss of dynamic range. One monkey was chosen to serve as the initial 
target (T0). Registration of the remaining 24 monkeys was initialized using manually identified homologous landmarks that included the center of the left and right eyeballs, the anterior commissure (AC), the posterior apex of the 4th ventricle as seen in a midline sagittal image, the most anterior aspect of the genu and the most posterior aspect of the corpus callosum, and the intersection of the central sulcus with the longitudinal fissure. All the monkeys were registered with a 7-parameter transformation (3 rotations, 3 translations, 1 scale), resampled and averaged in the space of T0 to form the initial average (A0). The center of the AC and posterior commissure (PC) were identified on A0 and used to define a transformation to bring the center of the $\mathrm{AC}$ to the origin $(0,0,0)$, align the $\mathrm{PC}$ along the negative $\mathrm{y}$-axis, and align the longitudinal fissure in the $\mathrm{x}=0$ plane and $\mathrm{A} 0$ was resampled onto a $0.25 \mathrm{~mm}$ isotropic grid to form Alin0.

After this initialization, the automatic cross-correlation based registration program minctracc (Collins et al., 1994) was used to compute the optimal 9-parameter transformation ( 3 translations, 3 rotations, 3 scales) that mapped each monkey to Alin0. Each MRI volume was resampled with the recovered transformations and averaged together to form Alin9. This process was repeated, estimating a 12-parameter transformation (3 translations, 3 rotations, 3 scales, 3 skews) to form Alin12. Afterwards, nonlinear registration was used to refine the average using a strategy based on that described by Grabner et al (2006) and similar to the DARTEL technique of Ashburner (2007). The ANIMAL algorithm (Collins et al., 1995) was used to estimate the nonlinear deformation field that best aligned local neighborhoods between each monkey volume and the average target. The nonlinear registration began by estimating a grid with $3 \mathrm{~mm}$ spacing. All MRI volumes were resampled through the recovered nonlinear 
transformation to form Anl3. The non-linear transformations were averaged together. The inverse of this average transformation was applied to Anl3 to remove any spatial bias from the previous target. This process was repeated twice, using a grid spacing of $2 \mathrm{~mm}$ with the Anl3 target to form the Anl2 target, and then using a grid spacing of $1 \mathrm{~mm}$ to form the Anl1 target. At this point, deformation fields were estimated to map all monkeys into the same space (Fig.1; Table 1). It is important to note that at each step, the transforms are concatenated so that the original data is re-sampled only once using a tricubic kernel to minimize interpolation errors.

\section{Transformation to cytoarchitecture atlas}

The average MNI monkey brain can be transformed into the stereotaxic space of the atlas of The Rhesus Monkey Brain in Stereotaxic Coordinates by Paxinos et al (2008) (see Chakravarty et al., 2008). Thus, the investigator can take advantage of the anatomical delineations of the cortical and subcortical structures defined on coronal histological sections of the atlas. There are three ways to transform anatomical T1 weighted macaque brain coordinates into the Paxinos et al. (2008) monkey atlas. The first two methods, which focus on mapping coordinates between the printed atlas and the MNI monkey space coordinate system, have been described before (Chakravarty et al., 2008) and are included in the supplementary information associated with this article. Briefly, both a linear and a nonlinear transformation are available to map coordinates from the MRI template to the Paxinos et al. (2008) printed atlas and vice versa using scripts that are provided on our web site. We offer the choice of referring to the MNI rhesus monkey space, the MNI cynomolgus monkey space, or the combined (rhesus and cynomolgus) MNI monkey space when performing the transformations. Furthermore, it is possible to 
register MNI monkey space volumes into the analysis software packages of SPM and FSL by initially converting the volume into either Analyze or Nifti file formats. The description and explanation of the steps for all of the above scripts can be found at (http://www.bic.mni.mcgill.ca/ServicesAtlases/HomePage).

\section{Results}

The average macaque brain

The average atlas comprises of $25 \mathrm{~T} 1$-weighted MRIs of normal young adult macaque brains. This atlas is constructed from the averaged position, orientation and scale of all the individual subjects and represents both the intensities and spatial positioning of the subjects' anatomical structures. The average MNI macaque monkey brain has superior contrast, signal and definition when compared to the individual macaque brain (Fig. 2). Note, in Figure 2, the greater contrast between gray and white matter as well as the improved delineation of the anatomical structures in the average MNI macaque monkey brain. The individual rhesus brain was transformed nonlinearly to the average macaque brain and the same contrast was used for each image set. The significant improvement in contrast and resolution of the average macaque brain demonstrates the high degree of alignment of the individual monkey brains. It should be noted that we maintained anatomical asymmetry between the left and right hemispheres in order to preserve left/right variability among the macaque population. This allows for the investigation of hemispheric differences and is more representative of the monkey population. The signalto-noise ratio (SNR) was calculated in homologous selected regions of interest (ROI) between the individual macaque brain and the average macaque volume. The results 
indicated that both the gray and white matter within the average volume had significantly greater SNR as compared to the individual brain. In addition the contrast to noise ratio (CNR) has been computed (Fig.2; Table 2). The greater clarity and definition of the cortical and subcortical structures in the average brain indicates the quality of alignment across all 25 subjects. Note that the signal intensity (bright region) located on the right medial orbital surface near the gyrus rectus is due to susceptibility artifacts between the edge of the brain and the tissue surrounding the eyeball (Fig. 2). The CNR of the average template was further estimated by measuring the mean and variance of the image intensity with manually defined ROIs in the gray matter and the white matter (Fig. 3). As expected, CNR increases with the number of animals averaged.

\section{MNI monkey space coordinate system}

A three dimensional stereotaxic coordinate system was placed onto the MNI average monkey brain (Fig.4). As in the stereotactic human brain atlas (Talairach and Tournoux, 1988), a line was defined in the mid-plane that passed through the superior aspect of the anterior commissure (AC) and the posterior aspect of the posterior commissure (PC). The origin of the space was defined by the intersection of the vertical line in the mid-plane, perpendicular to the $\mathrm{AC}-\mathrm{PC}$ line that passes through the posterior aspect of the $\mathrm{AC}(\mathrm{x}=0$, $\mathrm{y}=0, \mathrm{z}=0$ ). Positive $\mathrm{y}$-coordinates increase in the rostral direction while negative $\mathrm{y}-$ coordinates decrease in the caudal direction from AC. All coordinates are provided in millimeters. Note that this origin is slightly different from what is described by Chakravarty et al (2008) in the Paxinos et al. (2008) monkey atlas. An origin similar to the original human Talairach space (Talairach and Tournoux, 1988) was adopted in order 
to facilitate the transformation of our coordinate system to the Paxinos et al. (2008) space.

A significant advantage over the current atlases is that the MNI monkey atlas coordinates are obtained from data in vivo (i.e. the 3D digital MRI average of 25 live animals) and therefore not subject to shrinkage and deformations caused by perfusion, freezing and mounting artifacts of post-mortem brains

\section{Volumetric relationship of brain structures}

The segmentation of selected anatomical structures (see table 3) of the average macaque brain was done manually using the software package Display (MacDonald, 1996). In addition, each voxel in the average macaque brain was assigned a numerical value and was represented with a unique color in $3 \mathrm{D}$ (e.g. white matter $=1$, pink; gray matter $=2$, blue). A volumetric scaling factor was used to map the data from the animals' native space to the MNI monkey space in stereotaxic coordinates. The volume and standard deviation of these anatomical structures were collected and compared against the average brain volumes for each animal based on the number of voxels within a given label (Table 3). Two of the monkeys from the group were excluded from this volumetric study due to the fact that their temporal lobes were not fully captured in the MRI volume.

From the segmentation of the average macaque brain, volumetric information can be extracted automatically for most brain structures on individual brains using the ANIMAL non-linear registration and structure segmentation software (Collins and Evans, 1997) and visualized in 3D using the interactive Display software described above (Figs. 5, and Supplementary information). This volumetric information can be used to compare and 
contrast anatomical brain structures of individual monkey brains with the average macaque brain. The native volumes of each structure are also available by simply dividing the volume in space by the appropriate scaling factor for that animal.

\section{Deformation fields}

The deformation vector fields that non-linearly align each animal to the average MNI macaque monkey brain were averaged together and used to create the mean displacement map shown in Figure 6. The starting point for the non-linear deformation step was the last linear template Alin12. The displacement (color scale) represents the average distance that each voxel from each monkey brains had to be displaced (or warped) in order to match corresponding locations in the average macaque monkey brain. The deformations represent geometrical variations and not global differences such as brain size since all the monkey brains were registered with the reference brain through affine transformation. Larger voxel displacement is represented by the brighter colors (e.g., white) and corresponds to regions that have larger residual anatomical variability after affine mapping to the Alin12 space. Note that structures located in the center of the brain display the least amount of displacement and are known to have the least anatomical variability across subjects (Fig. 6).

A principal component analysis (PCA) was performed on the deformation data and the first, second and third components highlight the largest variations contained in the deformation fields. The largest variability was found bilaterally in the medial orbitofrontal cortex, the lateral visual cortex, centered on the lunate sulcus, and in the region of the spur of the arcuate sulcus. Interestingly, the two species of macaques 
(cynomolgus and rhesus) could be classified into their respective groups simply by using the discriminatory power in the eigenvalues.

\section{Comparison of cynomolgus and rhesus macaques}

In order to examine the similarities and differences in the morphology between the two species (cynomolgus and rhesus), two averages were created. For each species, the inverse of the average deformation for all members of that species was applied to the species average to remove the target bias. The two resulting volumes, one average rhesus (7 subjects) and one average cynomolgus (18 subjects) represent the best, unbiased average anatomy in the linear space of Alin12, and can be compared directly. For each average, the cortical pial surface was extracted using the marching cubes algorithm (Lorensen and Cline, 1987) applied to the manual skull masked average MRI volume with an isovalue of 50 (normalized between 0-100). The qualitative comparison of the 3D resulting surfaces revealed slight differences in the frontal pole, ventral posterior frontal cortex and in the temporal cortex (Fig. 7). The similarities and differences between the cynomolgus and rhesus monkeys were also evaluated using a deformation based morphology study of the nonlinear deformation fields (results not shown here). A composite $3 \mathrm{D}$ of both templates was also generated to reveal the slight differences in the anatomical structures of the two species. The full 3D results are available for viewing at (http://www.bic.mni.mcgill.ca/ServicesAtlases/HomePage). 


\section{Discussion:}

In the present investigation, 3D MRI volumes from a large group of macaque monkeys were aligned together to form an unbiased average brain. A stereotaxic coordinate system was fitted to this average brain to create the MNI monkey space. This digital stereotaxic coordinate system and atlas provides a number of advantages to the neuroscientist. The atlas will be useful to investigators of the anatomical correlates underlying function in awake nonhuman primates using classic electrophysiology and functional MRI (fMRI). The 3D stereotactic digital MNI monkey space will also be useful to researchers of the anatomy of the brain since data from individual animals can be normalized to the common space. This methodology will allow for better communication and presentation of results across laboratories in a standardized manner. Furthermore, any data described in the average standard MNI space can be matched (linearly or nonlinearly) to the histological delineations of cortical and subcortical structures of the Paxinos et al atlas (2008) (see Chakravarty et al., 2008, for more detail on the MRI-to-atlas transformation estimation procedure). Scripts have also been provided (http://www.bic.mni.mcgill.ca/ServicesAtlases/HomePage) for the fully automated transformation of individual MR monkey data sets into MNI monkey space or in stereotaxic coordinates based on the coordinates of the Paxinos et al (2008) atlas.

Our 3D MRI atlas exhibits several important advantages with respect to other digital macaque atlases (e.g. Black et al., 2001; McLaren et al., 2009; Vincent et al., 2007). First, our MRI macaque monkey atlas is based on a nonlinear average that can better communicate the spatial relationships and morphometry of the brain structures for aligning data. Our macaque atlas provides detailed structural segmentations to classify 
individual brain structures (i.e., temporal lobe structures). In addition, we offer separate nonlinear atlases for both the cynomolgus and the rhesus macaqueif the researcher prefers. Differences in skull shape are noticeable between the two types of monkeys (Fig. 6), and this fact translates into differences in the shape of the temporal and frontal poles in each species (differences in volumes for select structures between the two species have been placed in the Supplementary Information). However, the gross morphology and architecture of brain structures in the two species are very similar. Due to these similarities we felt comfortable to include a larger number of cynomolgus volumes in the average macaque template in order to improve on the signal to noise across the average. Second, references to anatomical structures and delineations from individual monkey volumes can be directly compared to a more detailed cytoarchitectonically comprehensive analysis of the monkey brain (Paxinos et al., 2008). Thirdly, we have quantified the anatomical variability of the animals that were used in our atlas. This is increasingly important for brain atlases in order to aid analyses, visualization and comparisons across subjects, time, and conditions. Fourthly, we have adopted an average coordinate system similar to that used in the human neuroimaging field (i.e., based on the anterior commissure) as the origin of reference for the digital atlas. Data from different studies can be directly compared if reported within this standard common stereotaxic space, as is the case with functional neuroimaging studies of the human brain (e.g., Talairach and Tournoux, 1988). Finally, we offer an automated method to normalize new T1 macaque monkey weighted data to our atlas space (http://www.bic.mni.mcgill.ca/ServicesAtlases/HomePage). This makes for a most 
convenient and automated way of visualizing individual macaque monkey coordinates in a digital format.

\section{References}

Ashburner, J., 2007. A fast diffeomorphic image registration algorithm. Neuroimage 38: 95-113.

Chakravarty, M., Frey, S., Collins, D.L., 2008. Digital atlas of the rhesus monkey brain in stereotaxic coordinates. In: Paxinos, G., Huang, X-F., Petrides, M., Toga, A.W. (Eds.), The Rhesus Monkey Brain in Stereotaxic Coordinates. Academic Press, San Diego, pp. 403-407.

Collins, D.L., Evans, A.C., 1997. ANIMAL: validation and applications of non-linear registration based segmentation. Int. J. Patttern Recogn. 11:1271-1294.

Collins, D.L., Neelin, P., Peters, T.M., Evans, A.C., 1994. Automatic 3D intersubject registration of MR volumetric data into standardized Talairach space. J. Comput. Assist. Tomogr. 18, 192-205.

Collins, D.L., Holmes, C.J., Peters, T., M., Evans, A.C., 1995. Automatic 3D modelbased neuroanatomical segmentation. Hum. Brain Map. 3:190-208.

Evans, A.C., Collins, D.L., Neelin, P., MacDonald, D., Kamber, M., Marrett, T.S., 1994a. Three-dimensional correlative imaging: Applications in human brain mapping. In Thatcher, R., Hallett, M., Zeffiro, T., John, E., Huerta, M. (Eds.), Advances in NeuroImaging: Multimodal Registration. Elsevier, NewYork, pp.145-162.

Evans, A.C., Kamber, M., Collins, D.L., MacDonald, D., 1994b. An MRI-based probabilistic atlas of neuroanatomy. In: Shorvon, S., Fish, D., Andermann, F., Bydder, G., Stefan, H. (Eds.), Magnetic Resonance Scanning and Epilepsy. Vol. 264 of NATO ASI Series A, Life Sciences, Plenum Press, New York, pp. 263-274.

Frey, S., Petrides, M., Collins, D.L., 2006. Macaque monkey brain templates. $12^{\text {th }}$ Annual Meeting of the Organization for Human Brain Mapping. NeuroImage Abstracts, 2006.

Frey, S., Comeau, R., Hynes, B., Mackey, S., Petrides, M., 2004. Frameless stereotaxy in the nonhuman primate. NeuroImage 23, 1226-1234. 
Frey, S., Pandya, D.N., Chakravarty, M.M., Petrides, M., Collins, D.L., 2009. MNI monkey space. Neuroscience Research, Vol. 65, Suppl. 1, pp. S130.

Grabner, G., Janke, A.L., Budge, M.M., Smith, D., Pruessner, J., Collins, D.L., 2006. Symmetric atlasing and model based segmentation: An application to the hippocampus in older adults. International Conference on Medical Image and Computer Assisted Intervention (MICCAI), pp. II-58, Springer, 2006.

Hampson, R.E., Pons, T.P., Stanford, T.R., Deadwyler, S.A., 2004. Categorization in the monkey hippocampus: a possible mechanism for encoding information into memory. Pros. Natl. Acad. Sci. USA 101, 3184-3189.

Lancaster, J.L., Tordesillas-Gutiérrez, D., Martinez, M., Salinas, F., Evans, A., Zilles, K., Mazziotta, J.C., Fox, P.T., 2007. Bias between MNI and Talairach coordinates analyzed using the ICBM-152 brain Template. Hum. Brain Map. 28, 1194-1205.

Lévesque, M., Parent, A., 2005. The striatofugal fiber system in primates: a reavaluation of its organization based on single-ax on tracing studies. Proc. Natl. Acad. Sci. USA 102, 11888-11893.

Lorensen, W.E., Cline, H.E., 1987. Marching cubes: a high resolution 3D surface construction algorithm. Comput. Graphics 21: 163-169.

Malkova, L., Gaffan, D., Murray, E.A., 1997. Excitotoxic lesions of the amygdala fail to produce impairment in visual learning for auditory secondary reinforcement but interfere with reinforcer devaluation effects in rhesus monkeys. J. Neurosci., 17, 6011-6020.

MacDonald, D. 1996. MNI-display: program for display and segmentation of surfaces and volumes. Retrieved May 27, 2009, from http://www.bic.mni.mcgill.ca/ServicesSoftwareVisualization/HomePage.

Martin, R.F., Bowden, D.M., 1996. A stereotaxic template atlas of the macaque brain for digital imaging and quantitative neuroanatomy. NeuroImage 4, 119-150.

Mazziotta, J., Toga, A., Evans, A., Fox, P., Lancaster, J., Zilles, K., Simpson, G., Woods, R., Paus, T., Pike, B., Holmes, C., Collins, L., Thompson, P., MacDonald, D., Schormann, T., Amunts, K., Palomero-Galagher, N., Parsons, L., Narr, K., Kabani, N., LeGoualher, G., Boomsma, D., Cnnon, T., Kawahima, R., Mazoyer, B., 2001. A probabilistic atlas and reference system for the human brain. Philos. Trans. R. Soc. Lond. B Biol. Sci. 356:1293-1322.

McLaren, D.G., Kosmatka, K.J., Oakes, T.R., Kroenke, C.D., Kohama, S.G., Matochik, J.A., Ingram, D.K., Johnson, S.C., 2009. A population-average MRI-based atlas collection of the rhesus macaque. Neuroimage 45, 52-59. 
Murray, E.A., Mishkin, M., 1998. Object recognition and location memory in monkeys with excitotoxic lesions of the amygdala and hippocampus. J. Neurosci., 18, 65686582 .

Paxinos, G., Huang, X-F., Toga, A.W., 2000. The Rhesus Monkey Brain in Stereotaxic Coordinates. Academic Press, San Diego.

Paxinos, G., Huang, X-F., Petrides, M., Toga, A.W., 2008. The Rhesus Monkey Brain in Stereotaxic Coordinates. Academic Press, San Diego.

Saleem, K., Logothetis, N., 2006. A Combined MRI and Histology Atlas of the Rhesus Monkey Brain. Elsevier, New York.

Saunders, R.C., Aigner, T.G., Frank, J.A., 1990. Magnetic resonance imaging of the rhesus monkey brain: use for stereotactic neurosurgery. Exp. Brain Res., 81, 443446.

Sled, JG., Zijdendbos, A.P., Evans, A.C., 1998. A nonparametric method for automatic correction of intensity nonuniformity in MRI data. IEEE Trans. Med. Imaging 17:87-97.

Szabo, J., Cowan, W.M., 1984. A Stereotaxic atlas of the brain of the cynomolgus monkey (Macaca fascicularis). J. Comp. Neurol. 222, 265-300.

Talairach, J., Tournoux, P., 1988. Co-planar Stereotaxic Altas of the Human Brain: 3Dimensional Proportional System: An Approach to Cerebral Imaging. Thieme, New York.

Van Essen, D.C., Dierker, D.L., 2007. Surface-based and probabilistic atlases of primate cerebral cortex. Neuron 56, 209-225.

Winters, W.D., Kado, R.T., Adey, W.R., 1969. A Stereotaxic Brain Atlas for Macaca Nemistrina. University of California Press, Berkeley. 


\section{Figure Legends}

Figure 1: Atlas creation. This figure shows the atlas at each successive step of the iterative processing with the evolving average template in the first row and the intensity standard deviation image in the second row. Note how anatomical detail is improved, and how the standard deviation image is reduced with each step of the atlas building process. A0; initial average; Alin; linear average; Anl; nonlinear average.

Figure 2: Comparison of an individual rhesus macaque brain (left) with the average macaque MNI atlas (right). The individual brain on the left was transformed nonlinearly to the average macaque brain and the same dynamic range was used for each image set to facilitate comparison. Note the greater contrast between gray and white matter as well as the improved delineation of the anatomical structures in the average brain. The significant improvement in contrast and resolution of the average macaque brain demonstrates the high degree of alignment of the individual monkey brains. The bright region to the right of the olfactory bulb is due to susceptibility artifacts. The ruler in the lower left axial slice indicates $1 \mathrm{~cm}$.

Figure 3: Averages of 1, 4, 8, 12, 16, 20 and 24 monkeys were created. White matter (WM): gray matter (GM) contrast to noise ratio (CNR) was estimated by measuring the mean and variance of the image intensity within a manually defined ROI in the caudate (for GM) and the corpus callosum (for WM). The CNR = ((mean (WM)mean $(\mathrm{GM})) /(\operatorname{sqrt}(\operatorname{var}(\mathrm{WM})+\operatorname{var}(\mathrm{GM})))$. As expected, CNR increases with the number of animals averaged, however, the last scans included were higher resolution and lower SNR creating a slight dip in the curve.

Figure 4: Stereotaxic coordinate system of the 3D MNI average monkey brain. As in the human Talairach and Tournoux atlas (1988), the superior posterior aspect of the anterior commissure was selected as the zero point for the MNI Monkey Atlas coordinate system $(0,0,0)$. The $\mathrm{x}$-axis (red) runs from left $(-)$ to right $(+)$. The $\mathrm{y}$-axis (green) runs from posterior (-) to anterior (+). The z-axis (blue) runs from inferior (-) to superior $(+)$. All coordinates are given in $\mathrm{mm}$.

Figure 5: Three-dimensional labeling of the atlas. The top row shows transverse slices through the MNI Monkey Atlas at $\mathrm{z}=-10,-5,0,5$, and $10 \mathrm{~mm}$. The bottom row shows superimposed labels on the transverse slices for the cortical gray and white matter, caudate, putamen, rhinal cortex, hippocampus, amygdala, globus pallidus, cerebellum, and corpus callosum.

Figure 6: Deformation vector fields were calculated for all the macaque monkey brains and averaged to form the mean displacement maps as seen above. The displacement (color scale) represents the average displacement (or warp) at each voxel that all monkey brains underwent in order to match corresponding locations in the average macaque monkey brain in $\mathrm{mm}$. The deformations represent local geometrical variations and not global differences such as brain size since all the monkey brains were registered with the reference brain through affine transformation. Larger voxel displacement is represented 
by the brighter colors (e.g., white). Note that structures located in the center of the brain display the least amount of displacement. The coordinates of the slices $(x=0, y=0, z=0)$.

Figure 7: Comparison of the cortical surface of the cynomolgus, (red) and the rhesus (green) macaque. Cortical surfaces were extracted from the average cynomolgus and the average rhesus macaque volumes in MNI monkey space. After normalization, the middle image demonstrates that the cortex of the rhesus macaque is slightly more rounded than the cynomolgus macaque, and the temporal lobes of the cynomolgus macaque extend more laterally in comparison to the rhesus macaque. 